\title{
THE CONTINUOUS WAVELET TRANSFORM AND WINDOW FUNCTIONS
}

\author{
J. N. PANDEY AND S. K. UPADHYAY
}

(Communicated by Ken Ono)

\begin{abstract}
We define a window function $\psi$ as an element of $L^{2}\left(\mathbb{R}^{n}\right)$ satisfying certain boundedness properties with respect to the $L^{2}\left(\mathbb{R}^{n}\right)$ norm and prove that it satisfies the admissibility condition if and only if the integral of $\psi\left(x_{1}, x_{2}, \cdots, x_{n}\right)$ with respect to each of the variables $x_{1}, x_{2}, \cdots, x_{n}$ along the real line is zero. We also prove that each of the window functions is an element of $L^{1}\left(\mathbb{R}^{n}\right)$. A function $\psi \in L^{2}\left(\mathbb{R}^{n}\right)$ satisfying the admissibility condition is a wavelet. We define the wavelet transform of $f \in L^{2}\left(\mathbb{R}^{n}\right)$ (which is a window function) with respect to the wavelet $\psi \in L^{2}\left(\mathbb{R}^{n}\right)$ and prove an inversion formula interpreting convergence in $L^{2}\left(\mathbb{R}^{n}\right)$. It is also proved that at a point of continuity of $f$ the convergence of our wavelet inversion formula is in a pointwise sense.
\end{abstract}

\section{INTRODUCTION}

The necessity for the inversion formula for the wavelet transform in dimensions higher than 1 has long been felt. Before the inversion formula for a two-dimensional wavelet transform was known, workers in image processing used to separate twodimensional wavelets into the product of two one-dimensional wavelets, thereby making the job very simple [12. But even two-dimensional wavelets are sometimes hard to separate and are sometimes impossible. Consider the wavelet $\psi\left(x_{1}, x_{2}\right)$ in $\mathbb{R}^{2}$ :

$$
\psi\left(x_{1}, x_{2}\right)=x_{1} x_{2} e^{-\left(x_{1}^{2}+1\right)\left(x_{2}^{2}+1\right)} \in S\left(\mathbb{R}^{2}\right) .
$$

This cannot be separated into the product of two one-dimensional wavelets so the use of the inversion formula for the wavelets in dimensions higher than 1 was needed by workers in image processing. Consider another wavelet in $\mathbb{R}^{2}$ given by

$$
\begin{aligned}
\psi\left(x_{1}, x_{2}\right) & = \begin{cases}x_{1} x_{2} e^{-\frac{1}{1-\left(x_{1}^{2}+x_{2}^{2}\right)}}, & |x|<1, \\
0, & |x| \geq 1,\end{cases} \\
|x| & =\sqrt{x_{1}^{2}+x_{2}^{2}}
\end{aligned}
$$

Received by the editors April 17, 2014 and, in revised form, July 2, 2014.

2010 Mathematics Subject Classification. Primary 46F12; Secondary 46F05, 46F10.

Key words and phrases. Continuous wavelet transform, Fourier inversion theory, inverse wavelet transform. 
This wavelet is a wavelet of compact support in $\mathbb{R}^{2}$ and cannot be separated. Now consider

$$
\psi(x)=\left\{\begin{array}{cl}
1, & 0 \leq x \leq \frac{1}{2} \\
-1, & \frac{1}{2}<x \leq 1 \\
0, & \text { elsewhere }
\end{array}\right.
$$

where $\psi(x)$ is a continuous wavelet. Using this wavelet we can define a two-dimensional wavelet of separable type by

$$
\psi\left(x_{1}, x_{2}\right)=\psi\left(x_{1}\right) \psi\left(x_{2}\right) .
$$

So one can work with the one-dimensional wavelet inversion formula in this case but not in the aforesaid previous two cases, as those are not the wavelets of separable type [12, p. 331].

Many authors worked on the inversion formula for the $n$-dimensional wavelet transform such as Daubechies [5], Meyer [7], Pathak [1], Keinert [6] and others. But the most notable amongst them are the works of Dauchechies [5, pp. 33-34] and Meyers [7, pp. 125-126].

We have proved the following wavelet inversion formula for $f \in L^{2}\left(\mathbb{R}^{n}\right)$. Our wavelet is a window function whose integral along each of the axes is zero, and our inversion formula is as follows:

$$
\frac{1}{C_{\psi}} \int_{\mathbb{R}^{n}} \int_{\mathbb{R}^{n}}|a|^{-1 / 2} \psi\left(\frac{x-b}{a}\right) W_{f}(a, b) \frac{d a d b}{|a|^{2}}=f,
$$

where

$$
W_{f}(a, b)=\frac{1}{\sqrt{|a|}}\left\langle f(t), \psi\left(\frac{t-b}{a}\right)\right\rangle
$$

and $|a|=\left|a_{1} \cdot a_{2} \cdots a_{n}\right|$. If we take $x=\left(x_{1}, x_{2}, \ldots, x_{n}\right), \Lambda=\left(\lambda_{1}, \lambda_{2}, \ldots, \lambda_{n}\right)$ and $|\Lambda|=\left|\lambda_{1} \cdot \lambda_{2} \cdots \lambda_{n}\right|$, then

$$
C_{\psi}=(2 \pi)^{n} \int_{\Lambda^{n}} \frac{|\hat{\psi}(\Lambda)|^{2}}{|\Lambda|} d \Lambda<\infty \quad \text { (admissibility condition), }
$$

where

$$
\hat{\psi}\left(\lambda_{1}, \lambda_{2}, \cdots, \lambda_{n}\right)=\hat{\psi}(\Lambda), \quad \text { the Fourier transform of } \psi(x) \in L^{2}\left(\mathbb{R}^{n}\right) .
$$

The convergence is interpreted in the $L^{2}\left(\mathbb{R}^{n}\right)$ sense and at a point of continuity of $f$ the convergence is in the pointwise sense. Note that in our derivation as shown later, we take $a=\left(a_{1}, a_{2}, \ldots, a_{n}\right)$ whereas Daubechies and Meyer take $a=(a, a, \ldots, a)$ and we do not choose $\psi$ as spherically symmetric. In our case $a_{1}, a_{2}, \ldots, a_{n}$ are all non-zero real numbers, whereas in their cases $a>0$.

We have used the Fourier inversion theorem to prove the wavelet inversion theorem. The advantage of this method is that it is simple and to prove the pointwise convergence only the continuity of $f$ is required and the continuity of the wavelet $\psi$ at a point $x=x_{0}$ concerned is not required. To prove the pointwise convergence using the Hilbert space technique, we require the continuity of the function $f$ and the wavelet $\psi$ both at a point $x=x_{0}$ concerned [4, p. 63]. It sounds quite strange but that is the way it is!

Our inversion formula is valid over $\mathbb{R}^{n} \times \mathbb{R}^{n}$ whereas the formulas derived by Daubechies and Meyer are valid only at $\mathbb{R} \times \mathbb{R}^{n}$. Thus our formula is more general than their inversion formula. 
We will give a characterisation of a subclass of functions belonging to $L^{2}\left(\mathbb{R}^{n}\right) \cap$ $L^{1}\left(\mathbb{R}^{n}\right)$ in the proof of the Corollary to Theorem 3.3 , which are wavelets. This makes the construction of wavelets useful to applied scientists much easier.

\section{Definitions AND PRELIMINARIES}

Definition 2.1. A function $f \in L^{2}\left(\mathbb{R}^{n}\right)$ is called a window function if it satisfies the following conditions:

(1) $x_{1} f, x_{2} f, \ldots, x_{n} f$ all belong to $L^{2}\left(\mathbb{R}^{n}\right)$.

(2) $x_{i} x_{j} f \in L^{2}\left(\mathbb{R}^{n}\right)$ for all $i, j=1,2, \ldots, n, i \neq j$.

(3) $x_{i} x_{j} x_{k} f \in L^{2}\left(\mathbb{R}^{n}\right)$ for all $i, j, k=1,2, \ldots, n, i \neq j \neq k \neq i$. Note that $i \neq j \neq k \neq i$ implies that $i, j, k$ are all different, $i \neq j \neq k$ may imply that $i$ and $k$ could be equal. Finally we have

(n) $x_{1} x_{2} \ldots x_{n} f \in L^{2}\left(\mathbb{R}^{n}\right)$.

Here, the lower suffixes in a term are all different. Let us illustrate this definition with reference to $n=2$. So if $f \in L^{2}\left(\mathbb{R}^{2}\right)$ we must have

$$
\begin{aligned}
& x_{1} f, x_{2} f \in L^{2}\left(\mathbb{R}^{2}\right), \\
& x_{1} x_{2} f \in L^{2}\left(\mathbb{R}^{2}\right) .
\end{aligned}
$$

Example 2.2. Define

$$
\psi(x)=\left\{\begin{array}{cl}
1, & 0 \leq x \leq \frac{1}{2}, \\
-1, & -\frac{1}{2}<x \leq 0, \\
0, & \text { elsewhere }
\end{array}\right.
$$

Then clearly $\psi$ is a window function in $L^{2}(\mathbb{R})$, and is a continuous wavelet.

Example 2.3. $\psi(x)=\left(x_{1}, x_{2} \ldots x_{n}\right) e^{-\left(x_{1}^{2}+x_{2}^{2}+\cdots+x_{n}^{2}\right)}$ is a window function in $L^{2}\left(\mathbb{R}^{n}\right)$, and is a wavelet.

The wavelet $\psi$ that we have chosen satisfies the following conditions:

(1) $\psi \in L^{2}\left(\mathbb{R}^{n}\right)$.

(2) $\psi$ is a window function as defined above.

(3) $\int_{-\infty}^{\infty} \psi(x) d x_{i}=0, \quad \forall i=1,2, \ldots, n$.

We will show that under the set of conditions (1), (2) and (3) the wavelet kernel $\psi$ satisfies the admissibility condition

$$
\int_{\Lambda^{n}} \frac{|\hat{\psi}(\Lambda)|^{2}}{|\Lambda|} d \Lambda<\infty,
$$

which enables us to prove the aforesaid wavelet inversion formula.

\section{Orthants AND PSEUdo-ORTHANTS}

Let $\mathbb{R}^{n}$ stand for the $n$-dimensional Euclidean space. Then $\mathbb{R}^{n}=\mathbb{R} \times \mathbb{R} \times \cdots \times \mathbb{R}$, $n$ times (Cartesian product):

$$
\begin{gathered}
\mathbb{R}^{n}=\prod_{i=1}^{n}\left[\mathbb{R}_{\left|x_{i}\right| \geq a} \cup \mathbb{R}_{\left|x_{i}\right| \leq a}\right], a>0, \\
\mathbb{R}_{\left|x_{i}\right| \geq a}=\left[x \in \mathbb{R}^{n}:\left|x_{i}\right| \geq a, x=\left(x_{1}, x_{2}, \ldots, x_{n}\right)\right],
\end{gathered}
$$


where $i=1,2, \ldots, n$.

Let $\triangle_{k}=\left[x:\left|x_{j_{1}}\right| \geq a,\left|x_{j_{2}}\right| \geq a, \ldots,\left|x_{j_{k}}\right| \geq a ;\left|x_{j_{k+1}}\right| \leq a,\left|x_{j_{k+2}}\right| \leq a, \ldots,\left|x_{j_{n}}\right|\right.$ $\leq a]$, where $j_{1}, j_{2}, \ldots, j_{n}$ are permutations of $1,2,3, \ldots, n ; \triangle_{k}$ is known as a pseudoorthant and when $a=0, \triangle_{k}$ is an orthant in $\mathbb{R}^{n}$.

Now $\mathbb{R}^{n}=\bigcup_{k=0}^{n}\left[\bigcup \triangle_{k}\right]$. Then the inner union has $\left(\begin{array}{l}n \\ k\end{array}\right)$ terms for a fixed $k \in$ $(0,1,2, \ldots, n)$. Varying $k$ from $0,1,2, \ldots, n$, we see that there are $\left(\begin{array}{l}n \\ 0\end{array}\right)+\left(\begin{array}{l}n \\ 1\end{array}\right)+\cdots+$ $\left(\begin{array}{l}n \\ n\end{array}\right)=2^{n}$ pseudo-orthants. The total number of pseudo-orthants in $\mathbb{R}^{n}$ can be easily figured out from the representation (3.1). There are $n$ factors in the representation (3.1) of $\mathbb{R}^{n}$ and there are two elements in each of the factors so there will be $2^{n}$ pseudo-orthants in $\mathbb{R}^{n}$.

From now on we will choose $a=1$ as there is no loss of generality in doing so.

Theorem 3.1. Let $f \in L^{2}\left(\mathbb{R}^{n}\right)$ be a window function on $\mathbb{R}^{n}$. Then $f \in L^{1}\left(\mathbb{R}^{n}\right)$.

Proof. By using Holder's inequality one can see that

$$
\begin{aligned}
\int_{\triangle_{k}} & |f(x)| d x \\
\quad & \int_{\triangle_{k}}\left|x_{j_{1}} x_{j_{2}} \cdots x_{j_{k}} f(x)\right| \frac{1}{\left|x_{j_{1}} x_{j_{2}} \cdots x_{j_{k}}\right|} d x \\
& \leq\left(\int_{\triangle_{k}}\left|x_{j_{1}} x_{j_{2}} \cdots x_{j_{k}} f(x)\right|^{2} d x\right)^{\frac{1}{2}}\left(\int_{\triangle_{k}} \frac{1}{\left|x_{j_{1}} x_{j_{2}} \cdots x_{j_{k}}\right|^{2}} d x\right)^{\frac{1}{2}} \\
& \leq\left(\int_{\mathbb{R}^{n}}\left|x_{j_{1}} x_{j_{2}} \cdots x_{j_{k}} f(x)\right|^{2} d x\right)^{\frac{1}{2}}\left(\left.\int_{\triangle_{k}} \frac{1}{\mid x_{j_{1}} x_{j_{2}} \cdots x_{j_{k}}}\right|^{2} d x\right)^{\frac{1}{2}} \\
& \leq\left\|x_{j_{1}} x_{j_{2}} \cdots x_{j_{k}} f(x)\right\|_{2}\left(\int_{\triangle_{k}} \frac{1}{x_{j_{1}}^{2} x_{j_{2}}^{2} \cdots x_{j_{k}}^{2}} d x\right)^{\frac{1}{2}} \\
& \leq\left\|x_{j_{1}} x_{j_{2}} \cdots x_{j_{k}} f\right\|_{2}\left(\left(\int_{-\infty}^{-1}+\int_{1}^{+\infty}\right) \frac{1}{x_{j_{1}}^{2}} d x_{j_{1}}\right)^{\frac{1}{2}} \\
& \times\left(\int_{-\infty}^{-1}+\int_{1}^{+\infty}\right) \frac{1}{x_{j_{2}}^{2}} d x_{j_{2}}^{\frac{1}{2}} \cdots\left(\int_{-\infty}^{-1}+\int_{1}^{+\infty}\right) \frac{1}{x_{j_{k}}^{2}} d x_{j_{k}}^{\frac{1}{2}} \\
& \times\left(\int_{-1}^{1} d x_{j_{k+1}} \int_{-1}^{1} d x_{j_{k+2}} \cdots \int_{-1}^{1} d x_{j_{k+n-k}}\right)^{\frac{1}{2}} \\
& \leq\left\|x_{j_{1}} x_{j_{2}} \cdots x_{j_{k}} f\right\|_{2} 2^{\frac{k}{2}} \cdot 2^{\frac{n-k}{2}} \\
& \leq 2^{\frac{n}{2}}\left\|x_{j_{1}} x_{j_{2}} \cdots x_{j_{k}} f\right\|_{2},
\end{aligned}
$$

and

$$
\int_{\bigcup \triangle_{k}}|f(x)| d x \leq 2^{\frac{n}{2}} \sum\left\|x_{j_{1}} x_{j_{2}} \cdots x_{j_{i}} \cdots x_{j_{k}} f\right\|_{2}
$$

where the number of terms in the R.H.S. summation is $\left(\begin{array}{l}n \\ k\end{array}\right)$.

Therefore,

$$
\begin{aligned}
\int_{\mathbb{R}^{n}}|f(x)| d x & =\sum_{k=0}^{n} \int_{\cup \triangle_{k}}|f(x)| d x \\
& \leq 2^{\frac{n}{2}}\left[\|f\|_{2}+\sum_{i=1}^{n}\left\|x_{i} f\right\|_{2}+\sum_{i, j=1, i \neq j}^{n}\left\|x_{i} x_{j} f\right\|_{2}+\cdots+\left\|x_{1} x_{2} \cdots x_{n} f\right\|_{2}\right] .
\end{aligned}
$$


Since $f$ is a window function, each of the terms in (3.2) is bounded. Therefore $\int_{\mathbb{R}^{n}}|f(x)| d x$ is bounded; i.e. $f \in L^{1}\left(\mathbb{R}^{n}\right)$. For the details of the proof one can see [10].

Corollary. Let $f \in L^{2}\left(\mathbb{R}^{n}\right)$ be a window function. Then $\hat{f}\left(\lambda_{1}, \lambda_{2}, \ldots, \lambda_{n}\right)$ is a continuous function of $\lambda_{1}, \lambda_{2}, \cdots, \lambda_{n}$, where $\hat{f}$ is the Fourier transform of $f$.

Note that Theorem 3.1 cannot be proved by breaking $\mathbb{R}^{n}$ into the unit sphere with the centre at the origin and a complement of it with respect to $\mathbb{R}^{n}$, hence there lies the importance of our window function technique.

Theorem 3.2. Let $f: \mathbb{R}^{n} \rightarrow C$ be a $L^{2}\left(\mathbb{R}^{n}\right)$ window function. Let $\hat{f}\left(\lambda_{1}, \lambda_{2}, \ldots, \lambda_{n}\right)$ be the Fourier transform of $f$ defined by

$$
\begin{aligned}
& \hat{f}\left(\lambda_{1}, \lambda_{2}, \ldots, \lambda_{n}\right) \\
& \quad=\frac{1}{(2 \pi)^{n / 2}} \int_{\mathbb{R}^{n}} f\left(x_{1}, x_{2}, \ldots, x_{n}\right) e^{-i\left(x_{1} \lambda_{1}+x_{2} \lambda_{2}+\cdots+x_{n} \lambda_{n}\right)} d x_{1} d x_{2} \ldots d x_{n} .
\end{aligned}
$$

Then the following two statements are equivalent.

(a) $\left.\hat{f}\left(\lambda_{1}, \lambda_{2}, \ldots, \lambda_{n}\right)\right|_{\lambda_{j}=0}=0$.

(b) $\int_{-\infty}^{\infty} f\left(x_{1}, x_{2}, \ldots, x_{j}, \ldots, x_{n}\right) d x_{j}=0, \quad j=1,2,3, \ldots$

Proof. The proof is very simple and so is omitted.

Plancherel formula ([3, p. 107] and [1, p. 75]). Suppose that $f$ and $g \in L^{2}$. Then $\langle F f, g\rangle=\langle f, F g\rangle,\langle F f, F g\rangle_{L^{2}}=\langle f, g\rangle_{L^{2}}$ and $\left\langle F^{-1} f, F^{-1} g\right\rangle_{L^{2}}=\langle f, g\rangle_{L^{2}}$. In particular

$$
\|F f\|_{L^{2}}=\|f\|_{L^{2}} .
$$

Here, $\langle u, v\rangle_{L^{2}}=\int_{-\infty}^{\infty} u(t) \overline{v(t)} d t . F, F^{-1}$ are defined below.

Fourier inversion theorem. Let $x=\left(x_{1}, x_{2}, \ldots, x_{n}\right)$ and $\omega=\left(\omega_{1}, \omega_{2}, \ldots, \omega_{n}\right)$ be elements of $\mathbb{R}^{n}$ and $f \in L^{2}\left(\mathbb{R}^{n}\right)$. Then

$$
F^{-1}(\hat{f})(x)=\underset{N \rightarrow \infty}{\lim .} \frac{1}{(2 \pi)^{n / 2}} \int_{-N}^{N} \hat{f}(\omega) e^{i \omega \cdot x} d \omega=f(x) .
$$

This defines convergence in $L^{2}\left(\mathbb{R}^{n}\right)$ and is called the limit in the mean (l.i.m.) [1, p. 75]. Here $\hat{f}(\omega)$ is the Fourier transform of $f$ defined by

$$
\hat{f}(\omega)=\underset{N \rightarrow \infty}{\lim } \frac{1}{(2 \pi)^{n / 2}} \int_{-N}^{N} f(x) e^{-i \omega \cdot x} d x,
$$

where $\omega \cdot x=\omega_{1} x_{1}+\omega_{2} x_{2}+\cdots+\omega_{n} x_{n}, N=\left(N_{1}, N_{2}, \ldots, N_{n}\right)$ and $N \rightarrow \infty$ implies that each of the components of $N$ tend to $\infty$ independently of each other. This is a well-known result, which was proved in several books such as [1,9]. At the point of continuity of $f$ the convergence in the above inversion formula is proved in the pointwise sense. The proof is given using the Plancherel formula

$$
\langle F f, F g\rangle=\langle f, g\rangle, \forall f, g \in L^{2}\left(\mathbb{R}^{n}\right),
$$


and replacing $g$ by the elements of the sequence $\left\{g_{m}(x)\right\}_{m=1}^{\infty}$ and letting $m \rightarrow \infty$; elements $g_{m}$ are defined as follows:

$$
\begin{aligned}
& g_{m}(x)=\left\{\begin{array}{l}
m^{n} \text { if }\left\|x_{m}-x_{0}\right\|_{1} \leq \frac{1}{2 m}, \\
0 \quad \text { if }\left\|x_{m}-x_{0}\right\|_{1}>\frac{1}{2 m},
\end{array}\right. \\
& x_{0}=\left(x_{0}^{1}, x_{0}^{2}, \ldots, x_{0}^{n}\right), \\
& \|x\|_{1}=\max \left(\left|x_{1}\right|,\left|x_{2}\right|, \cdots,\left|x_{n}\right|\right), \\
& x_{m}=\left(x_{0}^{1}+\frac{1}{2 m}, x_{0}^{2}+\frac{1}{2 m}, \cdots, x_{0}^{n}+\frac{1}{2 m}\right) .
\end{aligned}
$$

The norm \|\|$_{1}$ and $|x|=\sqrt{x_{1}^{2}+x_{2}^{2}+\cdots+x_{n}^{2}}=\|x\|$ generate the same topology over $\mathbb{R}^{n}$ as

$$
\|x\|_{1} \leq\|x\| \leq n\|x\|_{1} .
$$

The Fourier inversion formula $\frac{1}{(2 \pi)^{n / 2}} \int_{\mathbb{R}^{n}} F(\omega) e^{i \omega x_{0}} d w=f\left(x_{0}\right)$ with pointwise convergence at $x=x_{0}$ follows letting $m \rightarrow \infty$ in $\left\langle F f, F g_{m}\right\rangle=\left\langle f, g_{m}\right\rangle$. We assume that $f$ is continuous at $x_{0} \in \mathbb{R}^{n}$. Details of the proof are omitted and the proof is left as a simple and interesting exercise for the readers.

Admissibility condition. Let $f \in L^{2}\left(\mathbb{R}^{n}\right)$. We say that $f$ satisfies the admissibility condition if its Fourier transform $\hat{f}\left(\lambda_{1}, \lambda_{2}, \ldots, \lambda_{n}\right)$ satisfies the condition

$$
\int_{-\infty}^{\infty} \ldots \int_{-\infty}^{\infty} \int_{-\infty}^{\infty} \frac{\left|\hat{f}\left(\lambda_{1}, \lambda_{2}, \ldots, \lambda_{n}\right)\right|^{2}}{\left|\lambda_{1} \lambda_{2} \ldots \lambda_{n}\right|} d \lambda_{1} d \lambda_{2} \ldots d \lambda_{n}<\infty
$$

and then the function $f$ is said to be a basic wavelet.

We know that the Fourier transform $\hat{f}\left(\lambda_{1}, \lambda_{2}, \ldots, \lambda_{n}\right)$ of a window function $f$ is a continuous function of $n$ variables $\lambda_{1}, \lambda_{2}, \ldots, \lambda_{n}$. A window function belonging to $L^{2}\left(\mathbb{R}^{n}\right)$ is a regular (basic) wavelet function if it satisfies the admissibility condition

$$
\int_{\Lambda^{n}} \frac{\left|\hat{f}\left(\lambda_{1}, \lambda_{2}, \ldots, \lambda_{n}\right)\right|^{2}}{\left|\lambda_{1} \lambda_{2} \ldots \lambda_{n}\right|} d \lambda_{1} d \lambda_{2} \ldots d \lambda_{n}<\infty .
$$

So we see that $\hat{f}$ should be zero at each of the points $\left(0, \lambda_{1}, \lambda_{2}, \ldots, \lambda_{n}\right)$, $\left(\lambda_{1}, 0, \lambda_{2}, \ldots, \lambda_{n}\right), \ldots,\left(\lambda_{1}, \lambda_{2}, \ldots, \lambda_{n-1}, 0\right)$, i.e.

$$
\hat{f}\left(0, \lambda_{2}, \lambda_{3}, \ldots, \lambda_{n}\right), \hat{f}\left(\lambda_{1}, 0, \lambda_{3}, \lambda_{4}, \ldots, \lambda_{n}\right) \ldots
$$

and

By Theorem 3.2

$$
\hat{f}\left(\lambda_{1}, \lambda_{2}, \ldots, \lambda_{n-1}, 0\right) \text { are all zero. }
$$

$$
\begin{aligned}
& \hat{f}\left(0, \lambda_{2}, \lambda_{3}, \ldots, \lambda_{n}\right)=0 \Leftrightarrow \int_{-\infty}^{\infty} f\left(x_{1}, x_{2}, \ldots, x_{n}\right) d x_{1}=0, \\
& \hat{f}\left(\lambda_{2}, 0, \lambda_{3}, \ldots, \lambda_{n}\right)=0 \Leftrightarrow \int_{-\infty}^{\infty} f\left(x_{1}, x_{2}, \ldots, x_{n}\right) d x_{2}=0, \\
& \hat{f}\left(\lambda_{2}, \lambda_{3}, \ldots, \lambda_{n}, 0\right)=0 \Leftrightarrow \int_{-\infty}^{\infty} f\left(x_{1}, x_{2}, \ldots, x_{n}\right) d x_{n}=0 .
\end{aligned}
$$

If the window function $f$ is a regular (basic) wavelet, then it satisfies (3.4) $\Rightarrow$ $\Rightarrow(3.6)$. So we propose the following theorem. 
Theorem 3.3. Let $f \in L^{2}\left(\mathbb{R}^{n}\right)$ be a window function. Assume also that

$$
\int_{-\infty}^{\infty} f\left(x_{1}, x_{2}, \ldots, x_{i}, x_{n}\right) d x_{i}=0 \forall i=1,2, \ldots, n .
$$

Then $f$ satisfies the admissibility condition

$$
\int_{\Lambda^{n}} \frac{\left|\hat{f}\left(\lambda_{1}, \lambda_{2}, \ldots, \lambda_{n}\right)\right|^{2}}{\left|\lambda_{1} \lambda_{2} \ldots \lambda_{n}\right|} d \lambda_{1} d \lambda_{2} \cdots d \lambda_{n}<\infty .
$$

More precisely we have

$$
\begin{aligned}
& \int_{\Lambda^{n}} \frac{\left|\hat{f}\left(\lambda_{1}, \lambda_{2}, \ldots, \lambda_{n}\right)\right|^{2}}{\left|\lambda_{1} \lambda_{2} \ldots \lambda_{n}\right|} d \lambda_{1} d \lambda_{2} \ldots d \lambda_{n} \\
& \leq\left[\|f\|_{2}^{2}+2^{1} \sum_{i=1}^{n}\left\|x_{i} f\right\|_{2}^{2}+2^{2} \sum_{i, j=1, i \neq j}^{n}\left\|x_{i} x_{j} f\right\|_{2}^{2}\right. \\
& \left.\left.+2^{3} \sum_{\substack{i, j, k=1 \\
i \neq j \neq k \neq i}}^{\infty}\left\|x_{i} x_{j} x_{k} f\right\|_{2}^{2}+\cdots+2^{n} \| x_{1} x_{2} \ldots x_{n}\right) f \|_{2}^{2}\right] .
\end{aligned}
$$

Note that all the terms in the R.H.S. of (3.8) are bounded by virtue of the fact that $f$ is a window function.

Let us recall some notation as follows:

$$
\Lambda=\left(\lambda_{1}, \lambda_{2}, \lambda_{3}, \cdots, \lambda_{n}\right) \text { and }|\Lambda|=\left|\lambda_{1} \lambda_{2} \cdots \lambda_{n}\right| .
$$

$$
\hat{f}\left(\lambda_{1}, \lambda_{2}, \cdots, \lambda_{n}\right)=\hat{f}(\Lambda), \quad \text { the Fourier transform of } f(x) \in L^{2}\left(\mathbb{R}^{n}\right) .
$$

Here $x=\left(x_{1}, x_{2}, \ldots, x_{n}\right)$ and $\Lambda^{n}$ will stand for the $n$-dimensional Euclidean space where $\left(\lambda_{1}, \lambda_{2}, \cdots, \lambda_{n}\right)$ represents the coordinates of a point of it in general.

We also introduce the symbols $\Lambda_{0}, \Lambda_{1}, \Lambda_{2}, \cdots, \Lambda_{k}, \cdots, \Lambda_{n}$ as follows:

$\Lambda_{k}=\left[\left(\lambda_{1}, \lambda_{2}, \ldots, \lambda_{n}\right):\left|\lambda_{j_{i}}\right| \leq 1, i=1,2, \cdots, k ;\left|\lambda_{j_{i}}\right| \geq 1, i=k+1, k+2, \cdots, n\right]$, $\Lambda_{0}=\left[\left(\lambda_{1}, \lambda_{2}, \ldots, \lambda_{n}\right):\left|\lambda_{j_{i}}\right| \geq 1, i=1,2,3, \cdots, k, \cdots, n\right]$, $\Lambda_{n}=\left[\left(\lambda_{1}, \lambda_{2}, \ldots, \lambda_{n}\right):\left|\lambda_{j_{i}}\right| \leq 1, i=1,2,3, \cdots, n\right]$.

Taking all variations of suffixes of $\lambda_{j_{i}}$ we can see that $\Lambda_{k}$ can represent $\left(\begin{array}{l}n \\ k\end{array}\right)$ pseudo-orthants. The regions (pseudo-orthants) $\Lambda_{0}, \Lambda_{1}, \Lambda_{2}, \cdots, \Lambda_{k}, \cdots, \Lambda_{n}$ are all contained in $\Lambda^{n}$.

It is easy to show that

$$
\Lambda^{n}=\Lambda_{0} \cup\left(\bigcup \Lambda_{1}\right) \cup\left(\bigcup \Lambda_{2}\right) \cup \cdots \cup\left(\bigcup \Lambda_{k}\right) \cup \cdots \cup \Lambda_{n} .
$$

The symbol $d \Lambda$ stands for $d \lambda_{1} d \lambda_{2} \cdots d \lambda_{k} \cdots d \lambda_{n}$. Now

$$
\begin{aligned}
\int_{\Lambda^{n}} \frac{|\hat{f}(\Lambda)|^{2} d \Lambda}{|\Lambda|}= & \int_{\Lambda_{0}} \frac{|\hat{f}(\Lambda)|^{2}}{|\Lambda|} d \Lambda+\int_{\cup \Lambda_{1}} \frac{|\hat{f}(\Lambda)|^{2} d \Lambda}{|\Lambda|} \\
& +\int_{\cup \Lambda_{2}} \frac{|\hat{f}(\Lambda)|^{2}}{|\Lambda|} d \Lambda+\cdots+\int_{\Lambda_{n}} \frac{|\hat{f}(\Lambda)|^{2}}{|\Lambda|} d \Lambda \\
= & \int_{\left(\begin{array}{c}
n \\
0
\end{array}\right)=1 \text { term }}^{\Lambda_{0}} \frac{|\hat{f}(\Lambda)|^{2}}{|\Lambda|} d \Lambda+\sum \int_{\left.\begin{array}{c}
n \\
1 \\
1
\end{array}\right) \text { terms }}^{\Lambda_{1}} \frac{|\hat{f}(\Lambda)|^{2}}{|\Lambda|} d \Lambda
\end{aligned}
$$




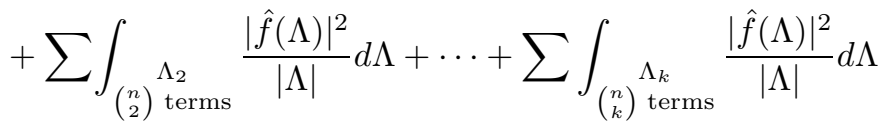

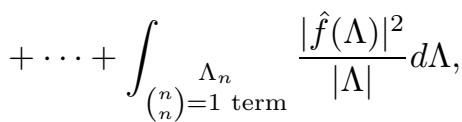

$$
\begin{aligned}
& \int_{\Lambda_{0}} \frac{|\hat{f}(\Lambda)|^{2}}{|\Lambda|} d \Lambda=\int_{\substack{i=1,2, \ldots, n \\
\left|\lambda_{i}\right| \geq 1}} \frac{|\hat{f}(\Lambda)|^{2}}{|\Lambda|} d \Lambda \leq \int_{\Lambda^{n}}|\hat{f}(\Lambda)|^{2} d \Lambda \\
& =\int_{\mathbb{R}^{n}}|f(x)|^{2} d x \\
& \left.=\|f\|_{2}^{2} \quad \text { [Plancherel's Theorem }\right] \text {. }
\end{aligned}
$$

So

$$
\int_{\Lambda_{0}} \frac{|\hat{f}(\Lambda)|^{2}}{|\Lambda|} d \Lambda \leq\|f\|_{2}^{2}
$$

Next we can find a bound for $\int_{\Lambda_{n}} \frac{|\hat{f}(\Lambda)|^{2}}{|\Lambda|} d \Lambda$ as follows. The function $\hat{f}\left(\lambda_{1}, \lambda_{2}, \cdots, \lambda_{i-1}, \lambda_{i}, \lambda_{i+1}, \cdots, \lambda_{n}\right)$ vanishes at points $\lambda_{i}=0, i=1,2,3, \cdots, n$. Therefore all the first partials of this function with respect to $\lambda_{1}, \lambda_{2}, \cdots, \lambda_{i-1}$, $\lambda_{i+1}, \cdots, \lambda_{n}$ vanish. Therefore,

$$
\hat{f}(\Lambda)=\int_{0}^{\lambda_{n}} \cdots \int_{0}^{\lambda_{2}} \int_{0}^{\lambda_{1}} \frac{\partial^{n} f(\Lambda)}{\partial \lambda_{1} \partial \lambda_{2} \cdots \partial \lambda_{n}} d \Lambda
$$

Now by Holder's inequality we have

$$
\begin{aligned}
&|\hat{f}(\Lambda)|^{2} \leq \int_{0}^{\lambda_{n}} \cdots \int_{0}^{\lambda_{2}} \int_{0}^{\lambda_{1}}\left|\frac{\partial^{n} \hat{f}(\Lambda)}{\partial \lambda_{1} \partial \lambda_{2} \cdots \partial \lambda_{n}}\right|^{2} d \Lambda\left|\int_{0}^{\lambda_{n}} \cdots \int_{0}^{\lambda_{2}} \int_{0}^{\lambda_{1}} 1^{2} d \Lambda\right|, \\
& \frac{|\hat{f}(\Lambda)|^{2}}{|\Lambda|} \leq \int_{0}^{\lambda_{n}} \cdots \int_{0}^{\lambda_{2}} \int_{0}^{\lambda_{1}}\left|\frac{\partial^{n} \hat{f}(\Lambda)}{\partial \lambda_{1} \partial \lambda_{2} \cdots \partial \lambda_{n}}\right|^{2} d \Lambda \\
& \leq \int_{\mathbb{R}^{n}}\left|\frac{\partial^{n} \hat{f}(\Lambda)}{\partial \lambda_{1} \partial \lambda_{2} \cdots \partial \lambda_{n}}\right|^{2} d \Lambda \\
& \leq \|\left. x_{1} x_{2} \cdots x_{n} f(x)\right|^{2} \\
& \text { [Plancherel's Theorem] }
\end{aligned}
$$

$$
\int_{\Lambda_{n}} \frac{|\hat{f}(\Lambda)|^{2}}{|\Lambda|} d \Lambda \leq\left\|x_{1} x_{2} \cdots x_{n} f(x)\right\|_{2}^{2} \times \int_{\Lambda_{n}} d \Lambda=\|x f(x)\|_{2}^{2} 2^{n} .
$$

We now proceed to find a bound for

$$
\begin{gathered}
\int_{\Lambda_{k}} \frac{|\hat{f}(\Lambda)|^{2}}{|\Lambda|} d \Lambda, \quad 1<k<n \\
\hat{f}(\Lambda)=\int_{0}^{\lambda_{j_{k}}} \int_{0}^{\lambda_{j_{k-1}}} \cdots \int_{0}^{\lambda_{j_{1}}} \frac{\partial^{k} \hat{f}(\Lambda)}{\partial \lambda_{j_{1}} \partial \lambda_{j_{2}} \partial \lambda_{j_{k}}} d \lambda_{j_{1}} d \lambda_{j_{2}} \cdots d \lambda_{j_{k}} .
\end{gathered}
$$


Using Holder's inequality we get

$$
\begin{aligned}
|\hat{f}(\Lambda)|^{2} \leq & \int_{0}^{\lambda_{j_{k}}} \cdots \int_{0}^{\lambda_{j_{2}}} \int_{0}^{\lambda_{j_{1}}}\left|\frac{\partial^{k} \hat{f}(\Lambda)}{\partial \lambda_{j_{1}} \partial \lambda_{j_{2}} \cdots \partial \lambda_{j_{k}}}\right|^{2} d \lambda_{j_{1}} d \lambda_{j_{2}} \cdots d \lambda_{j_{k}} \\
& \times\left|\int_{0}^{\lambda_{j_{k}}} \cdots \int_{0}^{\lambda_{j_{2}}} \int_{0}^{\lambda_{j_{1}}} 1^{2} d \lambda_{j_{1}} d \lambda_{j_{2}} \cdots d \lambda_{j_{k}}\right|
\end{aligned}
$$

So

$$
\begin{aligned}
& \frac{|\hat{f}(\Lambda)|^{2}}{\left|\lambda_{j_{1}} \lambda_{j_{2}} \cdots \lambda_{j_{k}}\right|} \\
& \quad \leq \int_{0}^{\lambda_{j_{k}}} \int_{0}^{\lambda_{j_{k-1}}} \cdots \int_{0}^{\lambda_{j_{2}}} \int_{0}^{\lambda_{j_{1}}}\left|\frac{\partial^{k} \hat{f}(\Lambda)}{\partial \lambda_{j_{1}} \partial \lambda_{j_{2}} \cdots \partial \lambda_{j_{k}}}\right|^{2} d \lambda_{j_{1}} d \lambda_{j_{2}} \cdots d \lambda_{j_{k}}, \\
& \frac{|\hat{f}(\Lambda)|^{2}}{\left|\lambda_{j_{1}} \lambda_{j_{2}} \cdots \lambda_{k} \lambda_{j_{k+1}} \cdots \lambda_{j_{n}}\right|} \\
& \quad \leq \int_{0}^{\lambda_{j_{k}}} \cdots \int_{0}^{\lambda_{j_{2}}} \int_{0}^{\lambda_{j_{1}}}\left|\frac{\partial^{k} \hat{f}(\Lambda)}{\partial \lambda_{j_{1}} \partial \lambda_{j_{2}} \cdots \partial \lambda_{j_{k}}}\right|^{2} d \lambda_{j_{1}} d \lambda_{j_{2}} \cdots d \lambda_{j_{k}}
\end{aligned}
$$

as $\left|\lambda_{j_{i}}\right| \geq 1$ for $i=k+1, k+2, \cdots, n$,

$$
\begin{aligned}
& \int \cdots \int \frac{|\hat{f}(\Lambda)|^{2}}{|\Lambda|} d \lambda_{j_{k+1}} d \lambda_{j_{k+2}} \cdots d \lambda_{j_{n}} \\
& \begin{array}{c}
\left|\lambda_{j_{i}}\right| \geq 1 \\
k+1, k+2, \cdots, n
\end{array} \\
& \leq \int_{0}^{\lambda_{j_{k}}} \int_{0}^{\lambda_{j_{k-1}}} \cdots \int_{0}^{\lambda_{j_{2}}} \int_{0}^{\lambda_{j_{1}}} \quad \int_{\left|\lambda_{j_{i}}\right| \geq 1} \ldots \int_{\mid}\left|\frac{\partial^{k} \hat{f}(\Lambda)}{\partial \lambda_{j_{1}} d \lambda_{j_{2}} \cdots \partial \lambda_{j_{k}}}\right|^{2} d \Lambda \\
& \leq\left\|\frac{\partial^{k} \hat{f}(\Lambda)}{\partial \lambda_{j_{1}} \partial_{j_{2}} \cdots \partial \lambda_{j_{k}}}\right\|_{2}^{2} \\
& (3.12) \leq\left\|x_{j_{1}} x_{j_{2}} \cdots x_{j_{k}} f(x)\right\|_{2}^{2}, \\
& \int \cdots \int \quad \int \cdots \int \frac{|\hat{f}(\Lambda)|^{2}}{|\Lambda|} d \lambda_{j_{1}} d \lambda_{j_{2}} \cdots d \lambda_{j_{k}} d \lambda_{j_{k+1}} d \lambda_{j_{k+2}} \cdots d \lambda_{j_{n}} \\
& \begin{array}{cc}
\left|\lambda_{j_{i}}\right| \leq 1 & \left|\lambda_{j_{i}}\right| \geq 1 \\
i=1,2,3, \cdots, k & i=k+1, k+2, \cdots, n
\end{array} \\
& \leq\left\|x_{j_{1}} x_{j_{2}} \cdots x_{j_{k}} f(x)\right\|_{2}^{2} \int_{\substack{\left|\lambda_{i}\right| \leq 1 \\
i=1,2, \cdots, k}} \cdots \int_{j_{1}} d \lambda_{j_{2}} \cdots d \lambda_{j_{k}}, \\
& \int_{\Lambda_{k}} \frac{|\hat{f}(\Lambda)|^{2}}{|\Lambda|} d \Lambda \leq 2^{k}\left\|x_{j_{1}} x_{j_{2}} \cdots x_{j_{k}} f(x)\right\|_{2}^{2} .
\end{aligned}
$$


Now using (3.9) and the other foregoing results, we get

$$
\begin{gathered}
\int_{\Lambda^{n}} \frac{|\hat{f}(\Lambda)|^{2}}{|\Lambda|} d \Lambda \leq \\
+\cdots f(x)\left\|_{2}^{2}+2 \sum_{i=1}^{n}\right\| x_{i} f(x)\left\|_{2}^{2}+2^{2} \sum_{\substack{i, j=1 \\
i \neq j}}^{n}\right\| x_{i} x_{j} f(x) \|_{2}^{2} \\
+\cdots+2^{n}\left\|x_{1} x_{2} \cdots x_{n} f(x)\right\|_{2}^{2} \cdot
\end{gathered}
$$

Corollary. Let $f \in L^{2}\left(\mathbb{R}^{n}\right)$ be a window function. Then $f$ satisfies the admissibility condition if and only if $\int_{-\infty}^{\infty} f\left(x_{1}, x_{2}, \ldots, x_{i}, \ldots, x_{n}\right) d x_{i}=0, \quad i=1,2, \ldots, n$.

Proof. Sufficiency follows from Theorem 3.3. The necessity follows by virtue of the fact that if $f$ is a window function, then its Fourier transform is a continuous function of $\lambda_{1}, \lambda_{2}, \ldots, \lambda_{n}$. Therefore $\hat{f}$ must vanish at any point in $\lambda$-coordinates whose one component is zero to maintain the admissibility condition (3.7).

$$
\begin{aligned}
\int_{-\infty}^{\infty} f\left(x_{1}, x_{2}, \ldots, x_{i} \ldots, x_{n}\right) d x_{i}=0 & \\
& \Leftrightarrow \hat{f}\left(\lambda_{1}, \lambda_{2}, \ldots, \lambda_{i-1}, 0, \lambda_{i+1}, \ldots, \lambda_{n}\right)=0, \lambda_{i}=0 \forall i=1,2, \ldots, n .
\end{aligned}
$$

Examples of window functions which are wavelets. $t e^{-|t|}, \frac{t}{1+|t|^{3}}, \frac{t}{1+t^{4}}$ in $L^{2}(\mathbb{R}), \prod_{i=1}^{n} t_{i} e^{-t_{i}^{2}}, \prod_{i=1}^{n} \frac{t_{i}}{1+t_{i}^{4}}$, in $L^{2}\left(\mathbb{R}^{n}\right)$.

\section{WAVELET INVERSiON FORMULA FOR FUNCTIONS IN $L^{2}\left(\mathbb{R}^{n}\right)$}

Let $\psi \in L^{2}\left(\mathbb{R}^{n}\right)$ be a window function satisfying the additional conditions $\int_{-\infty}^{\infty} \psi\left(x_{1}, x_{2}, x_{3}, \ldots, x_{i}, \ldots, x_{n}\right) d x_{i}=0, \quad i=1,2,3, \ldots, n$. Then $\psi$ is called a regular or basic wavelet function since it then also satisfies the admissibility condition

$$
\int_{-\infty}^{\infty} \ldots \int_{-\infty}^{\infty} \int_{-\infty}^{\infty} \frac{\left|\hat{\psi}\left(\lambda_{1}, \lambda_{2}, \lambda_{3}, \ldots, \lambda_{n}\right)\right|^{2}}{\left|\lambda_{1} \lambda_{2} \ldots \lambda_{n}\right|} d \lambda_{1} d \lambda_{2} \ldots d \lambda_{n}<\infty
$$

Let $\hat{\psi}$ be the Fourier transform of $\psi \in L^{2}\left(\mathbb{R}^{n}\right)$ as described above and let $C_{\psi}=$ $(2 \pi)^{n} \int_{\mathbb{R}^{n}} \frac{\left|\hat{\psi}\left(\lambda_{1}, \lambda_{2}, \lambda_{3}, \ldots, \lambda_{n}\right)\right|^{2}}{\left|\lambda_{1} \lambda_{2} \ldots \lambda_{n}\right|} d \lambda_{1} d \lambda_{2} \ldots d \lambda_{n}$. The integral in one dimension of a function $f$ on $\mathbb{R}$ can be written as

$$
\int_{-\infty}^{\infty} f(x) d x=\int_{-\mathbf{N}}^{\mathbf{M}} f(x) d x+\int_{\mathbf{M}}^{\infty} f(x) d x+\int_{-\infty}^{-\mathbf{N}} f(x) d x
$$

provided the integral of $f(x)$ on the real line is convergent. So

$$
\int_{-\infty}^{\infty} f(x) d x=\int_{-\mathbf{N}}^{\mathbf{M}} f(x) d x+R(M,-N),
$$

where $R(M,-N)$ is the remainder term. If $f(x, y)$ is integrable over the $X Y$-plane, then

$$
\int_{-\infty}^{\infty} \int_{-\infty}^{\infty} f(x, y) d x d y=\int_{-N_{2}}^{M_{2}} \int_{-N_{1}}^{M_{1}} f(x, y)+R\left(M_{1}, M_{2},-N_{1},-N_{2}\right) .
$$


The remainder term $R\left(M_{1}, M_{2},-N_{1},-N_{2}\right)$ can be represented by $R(\mathbf{M},-\mathbf{N})$ for simplicity and it can be worked out that $R(\mathbf{M},-\mathbf{N})=$ the sum of eight integrals and if $f(x, y) \in L\left(\mathbb{R}^{2}\right)$, then $R(\mathbf{M},-\mathbf{N}) \rightarrow 0$ as $\mathbf{M}, \mathbf{N} \rightarrow \infty$ independently of each other. If $f \in L\left(\mathbb{R}^{n}\right)$ the integral

$$
\int_{\mathbb{R}^{n}} f(x) d x=\int_{-N_{n}}^{M_{n}} \cdots \int_{-N_{2}}^{M_{2}} \int_{-N_{1}}^{M_{1}} f(x) d x+R(\mathbf{M},-\mathbf{N}) .
$$

The remainder term $R(\mathbf{M},-\mathbf{N})$ consists of finitely many, i.e. $\left(3^{n}-1\right)$, integrals and the remainder $R(\mathbf{M},-\mathbf{N}) \rightarrow 0$ as $M_{1}, M_{2}, \ldots, M_{n}, N_{1}, N_{2}, \ldots, N_{n}$ all go to infinity independently of each other. We will make use of this fact in proving the following.

Theorem 4.1. Let $f \in L^{2}\left(\mathbb{R}^{n}\right)$ and define the wavelet transform of $f$ by

$$
\begin{aligned}
& W_{f}\left(b_{1}, b_{2}, \ldots, b_{n}, a_{1}, a_{2}, \ldots, a_{n}\right) \\
& \quad=\left\langle f\left(x_{1}, x_{2}, \ldots, x_{n}\right), \frac{1}{\sqrt{\left|a_{1} a_{2} \ldots a_{n}\right|}} \psi\left(\frac{x_{1}-b_{1}}{a_{1}}, \frac{x_{2}-b_{2}}{a_{2}}, \ldots, \frac{x_{n}-b_{n}}{a_{n}}\right)\right\rangle,
\end{aligned}
$$

where $\psi$ is a basic wavelet as defined above in Section 2, i.e. $\psi$ is a window function satisfying

$$
\int_{-\infty}^{\infty} \psi(x) d x_{i}=0 \quad \forall i=1,2, \ldots n .
$$

Let $F_{b_{1}, b_{2}, \ldots, b_{n}}$ be the Fourier transform operator with respect to variables $b_{1}, b_{2}$, $\ldots, b_{n}$. Then

$$
\begin{aligned}
& F_{b_{1}, b_{2}, \ldots, b_{n}} W_{f}\left(b_{1}, b_{2}, \ldots, b_{n}, a_{1}, a_{2}, \ldots, a_{n}\right) \\
& \quad=\left\langle f\left(x_{1}, x_{2}, \ldots, x_{n}\right), \bar{F}_{b_{1}, b_{2}, \ldots, b_{n}} \frac{\psi\left(\frac{x_{1}-b_{1}}{a_{1}}, \frac{x_{2}-b_{2}}{a_{2}}, \ldots, \frac{x_{n}-b_{n}}{a_{n}}\right)}{\sqrt{\left|a_{1} a_{2} \ldots a_{n}\right|}}\right\rangle .
\end{aligned}
$$

Proof.

$$
\begin{aligned}
& F_{x_{1}, x_{2}, \ldots, x_{n}} \psi\left(\frac{x_{1}-b_{1}}{a_{1}}, \frac{x_{2}-b_{2}}{a_{2}}, \ldots, \frac{x_{n}-b_{n}}{a_{n}}\right)\left(\lambda_{1}, \lambda_{2}, \ldots, \lambda_{n}\right) \\
& =\left|a_{1} a_{2} \ldots a_{n}\right| \hat{\psi}\left(a_{1} \lambda_{1}, a_{2} \lambda_{2}, \ldots, a_{n} \lambda_{n}\right) e^{-i\left(\lambda_{1} b_{1}+\lambda_{2} b_{2}+\cdots+\lambda_{n} b_{n}\right)}
\end{aligned}
$$

and

$$
\begin{aligned}
& \left\langle f\left(x_{1}, x_{2}, \ldots, x_{n}\right), \psi\left(\frac{x_{1}-b_{1}}{a_{1}}, \frac{x_{2}-b_{2}}{a_{2}}, \ldots, \frac{x_{n}-b_{n}}{a_{n}}\right)\right\rangle \\
& =\left\langle F f\left(x_{1}, x_{2}, \ldots, x_{n}\right), F \psi\left(\frac{x_{1}-b_{1}}{a_{1}}, \frac{x_{2}-b_{2}}{a_{2}}, \ldots, \frac{x_{n}-b_{n}}{a_{n}}\right)\right\rangle \\
& \quad[\text { by Plancherel's lemma }] \\
& =\left\langle\hat{f}\left(\lambda_{1}, \lambda_{2}, \ldots, \lambda_{n}\right), \hat{\psi}\left(a_{1} \lambda_{2}, a_{2} \lambda_{2}, \ldots, a_{n} \lambda_{n}\right)\left|a_{1} a_{2} \ldots a_{n}\right|\right. \\
& \left.\times e^{-i\left(\lambda_{1} b_{1}+\lambda_{2} b_{2}+\cdots+\lambda_{n} b_{n}\right)}\right\rangle \\
& =\left|a_{1} a_{2} \ldots a_{n}\right| \int_{\mathbb{R}^{n}} \hat{f}\left(\lambda_{1}, \lambda_{2}, \ldots, \lambda_{n}\right) \overline{\hat{\psi}}\left(a_{1} \lambda_{1}, \ldots, a_{n} \lambda_{n}\right) \\
& \times e^{i\left(\lambda_{1} b_{1}+\lambda_{2} b_{2}+\cdots+\lambda_{n} b_{n}\right)} d \lambda_{1} d \lambda_{2} \ldots d \lambda_{n},
\end{aligned}
$$


where $\hat{\psi}\left(\lambda_{1}, \lambda_{2}, \ldots, \lambda_{n}\right)$ is bounded uniformly on $\mathbb{R}^{n}$ and $\hat{f} \in L^{2}\left(\mathbb{R}^{n}\right)$, so $\hat{f} \overline{\hat{\psi}} \in$ $L_{\lambda_{1}, \lambda_{2}, \ldots, \lambda_{n}}^{2}$. Therefore the above integral in (4.2) belongs to $L_{b_{1}, b_{2}, \ldots, b_{n}}^{2}$ by the property of the inverse Fourier transform. Now let

$$
\begin{gathered}
I=\int_{\mathbb{R}^{n}} f\left(x_{1}, x_{2}, \ldots, x_{n}\right) \bar{\psi}\left(\frac{x_{1}-b_{1}}{a_{1}}, \frac{x_{2}-b_{2}}{a_{2}}, \ldots, \frac{x_{n}-b_{n}}{a_{n}}\right) d x_{1} d x_{2} \ldots d x_{n} \\
=\int_{-N_{n}}^{M_{n}} \int_{-N_{n-1}}^{M_{n-1}} \ldots \int_{\mathbf{N}_{2}}^{M_{2}} \int_{-N_{1}}^{M_{1}} f\left(x_{1}, x_{2}, \ldots, x_{n}\right) \\
\quad \times \bar{\psi}\left(\frac{x_{1}-b_{1}}{a_{1}}, \frac{x_{2}-b_{2}}{a_{2}}, \ldots, \frac{x_{n}-b_{n}}{a_{n}}\right) d x_{1} d x_{2} \ldots d x_{n} \\
+R(M,-N, b) \\
=\int_{-\mathbf{N}}^{\mathbf{M}} f(\mathbf{x}) \bar{\psi}\left(\frac{\mathbf{x}-\mathbf{b}}{\mathbf{a}}\right) d \mathbf{x}+R(\mathbf{M},-\mathbf{N}, \mathbf{b}) \\
=I(\mathbf{M},-\mathbf{N}, \mathbf{b})+R(\mathbf{M},-\mathbf{N}, \mathbf{b}), \\
F_{\mathbf{b}} I=F_{\mathbf{b}} I(\mathbf{M},-\mathbf{N}, \mathbf{b})+F_{\mathbf{b}} R(\mathbf{M},-\mathbf{N}, \mathbf{b}) \\
=\int_{-\mathbf{N}}^{\mathbf{M}} f(\mathbf{x}) F_{\mathbf{b}} \bar{\psi}\left(\frac{\mathbf{x}-\mathbf{b}}{\mathbf{a}}\right) \mathbf{d x} \\
+F_{\mathbf{b}} R(\mathbf{M},-\mathbf{N}, \mathbf{b}) \quad[\text { by Fubini's theorem]. }
\end{gathered}
$$

$R(\mathbf{M},-\mathbf{N}, \mathbf{b})$ as a function of $\mathbf{b}$ goes to zero in $L^{2}\left(\mathbb{R}^{n}\right)$ as $\mathbf{M}, \mathbf{N} \rightarrow \infty$ independently of each other; therefore $F_{\mathbf{b}} R(\mathbf{M},-\mathbf{N}, \mathbf{b})$ also goes to zero in $L^{2}\left(\mathbb{R}^{n}\right)$ as a function of $\boldsymbol{\Lambda}$. Hence, letting $\mathbf{M}, \mathbf{N} \rightarrow \infty$ in (4.4) we obtain the desired result. The desired result could have been obtained by switching the order of integration in $\mathbf{F}_{\mathbf{b}} \mathbf{I}$, but we did not do so as neither of the iterated integrals $\int_{\mathbb{R}^{n}} \int_{\mathbb{R}^{n}} f(\mathbf{x}) F_{\mathbf{b}} \bar{\psi}\left(\frac{\mathbf{x}-\mathbf{b}}{\mathbf{a}}\right) d \mathbf{x} d \mathbf{b}$ and $\int_{\mathbb{R}^{n}} \int_{\mathbb{R}^{n}} f(\mathbf{x}) F_{\mathbf{b}} \bar{\psi}\left(\frac{\mathbf{x}-\mathbf{b}}{\mathbf{a}}\right) d \mathbf{b} d \mathbf{x}$ is absolutely convergent, nor is there any other way of proving that the double integral exists. So we applied Fubini's theorem as shown above in (4.3).

Theorem 4.2. Let $\psi$ be a basic or regular wavelet function in $L^{2}\left(\mathbb{R}^{n}\right)$ satisfying the admissibility condition (3.1) and assume that $f \in L^{2}\left(\mathbb{R}^{n}\right)$. Then the following inversion formula holds:

$$
\begin{aligned}
f(x)=\frac{1}{C_{\psi}} & \int_{\mathbb{R}^{n}}\left|a_{1} . a_{2} \ldots \ldots a_{n}\right|^{-1 / 2} \psi\left(\frac{x_{1}-b_{1}}{a_{1}}, \frac{x_{2}-b_{2}}{a_{2}}, \ldots, \frac{x_{n}-b_{n}}{a_{n}}\right) \\
& \times W_{f}\left(a_{1}, a_{2}, \ldots, a_{n}, b_{1}, b_{2}, \ldots, b_{n}\right) \frac{d b_{1} d b_{2} \ldots d b_{n} \ldots d a_{1} d a_{2} \ldots d a_{n}}{\left|a_{1} a_{2} \ldots a_{n}\right|^{2}},
\end{aligned}
$$

where $W_{f}\left(a_{1}, a_{2}, \ldots, a_{n}, b_{1}, b_{2}, \ldots, b_{n}\right)$ is defined to be the integral wavelet transform of $f \in L^{2}\left(\mathbb{R}^{n}\right)$ by

$$
\begin{aligned}
\int_{\mathbb{R}^{n}}\left|a_{1} . a_{2} \ldots \ldots a_{n}\right|^{-1 / 2} f( & \left.x_{1}, x_{2}, \ldots, x_{n}\right) \\
& \times \bar{\psi}\left(\frac{x_{1}-b_{1}}{a_{1}}, \frac{x_{2}-b_{2}}{a_{2}}, \ldots, \frac{x_{n}-b_{n}}{a_{n}}\right) d x_{1} d x_{2} \ldots d x_{n} .
\end{aligned}
$$

The $a_{i}$ 's and $b_{i}$ 's are real numbers, and the $a_{i}$ 's are non-zero. When $a_{i}$ is zero the above wavelet transform of $f$ is defined to be zero. In duality notation we write it 
in the form

$$
\begin{aligned}
W_{f}\left(a_{1}, a_{2}, \ldots, a_{n}, b_{1}, b_{2}, \ldots, b_{n}\right) \\
\quad=\left\langle f\left(x_{1}, x_{2}, \ldots, x_{n}\right), \frac{\psi\left(\frac{x_{1}-b_{1}}{a_{1}}, \frac{x_{2}-b_{2}}{a_{2}}, \ldots, \frac{x_{n}-b_{n}}{a_{n}}\right)}{\sqrt{\left|a_{1} a_{2} \ldots a_{n}\right|}}\right\rangle .
\end{aligned}
$$

The inversion formula (4.5) is proved interpreting convergence in $L^{2}\left(\mathbb{R}^{n}\right)$; at the point of continuity of $f$ the convergence in (4.5) takes place in the pointwise sense, as indicated in section 3 immediately preceding 3.3 .

Proof. Our proof will be sketchy, leaving the verification of the lengthy computations to the readers.

Step A. In the one-dimensional case the proof of this step is given in the book of Bogess and Narcovich [3. This result is

$$
F_{b}\left(\bar{\psi}\left(\frac{x-b}{a}\right)\right)=|a| e^{-i \lambda x}[\overline{\hat{\psi}(a \lambda)}] .
$$

Therefore,

$$
\overline{F_{b} \bar{\psi}\left(\frac{x-b}{a}\right)(\lambda)}=|a| e^{i \lambda x} \hat{\psi}(a \lambda) .
$$

In the $n$-dimensional case using substitutions $\frac{x_{i}-b_{i}}{a_{i}}=v_{i}, i=1,2,3, \ldots, n$, and the same trick of calculation as used by Bogess and Narcovich to prove (4.5), we get

$$
\begin{aligned}
& F_{b_{1}, b_{2}, \ldots, b_{n}} \bar{\psi}\left(\frac{x_{1}-b_{1}}{a_{1}}, \frac{x_{2}-b_{2}}{a_{2}}, \ldots, \frac{x_{n}-b_{n}}{a_{n}}\right) \\
& =\left|a_{1} a_{2} \ldots a_{n}\right| e^{-i\left(\lambda_{1} x_{1}+\lambda_{2} x_{2}+\cdots+\lambda_{n} x_{n}\right)} \overline{\hat{\psi}}\left(a_{1} \lambda_{1}, a_{2} \lambda_{2}, \ldots, a_{n} \lambda_{n}\right) .
\end{aligned}
$$

Step B. So, using a similar technique we get

$$
F_{b_{1}, b_{2}, \ldots, b_{n}}\left\{W_{f}\left(a_{1}, a_{2}, \ldots, a_{n}, b_{1} b_{2}, \ldots, b_{n}\right)\right\}=\prod_{i=1}^{n} \sqrt{2 \pi\left|a_{i}\right|} \overline{\hat{\psi}} \hat{f}\left(\lambda_{1}, \lambda_{2}, \ldots, \lambda_{n}\right) .
$$

Here the wavelet transform of $f$ is defined as $W_{f}: \mathbb{R}^{n} \times \mathbb{R}^{n} \rightarrow C$ :

$$
\begin{aligned}
& W_{f}\left(a_{1}, a_{2}, \ldots, a_{n}, b_{1}, b_{2}, \ldots, b_{n}\right) \\
& =\int_{\mathbb{R}^{n}} \frac{f\left(x_{1}, x_{2}, \ldots, x_{n}\right)}{\sqrt{\left|a_{1} a_{2} \ldots a_{n}\right|}} \bar{\psi}\left(\frac{x_{1}-b_{1}}{a_{1}}, \frac{x_{2}-b_{2}}{a_{2}}, \ldots, \frac{x_{n}-b_{n}}{a_{n}}\right) d x_{1} d x_{2} \ldots d x_{n}
\end{aligned}
$$

and we also use the fact proved in the previous theorem that

$$
\begin{aligned}
& F_{b_{1}, b_{2}, \ldots, b_{n}} W_{f}\left(a_{1}, a_{2}, \ldots, a_{n}, b_{1}, b_{2}, \ldots, b_{n}\right) \\
& =\int_{\mathbb{R}^{n}} \frac{f\left(x_{1}, x_{2}, \ldots, x_{n}\right)}{\sqrt{\left|a_{1} a_{2} \ldots a_{n}\right|}} F_{b_{1}, b_{2}, \ldots, b_{n}} \bar{\psi}\left(\frac{x_{1}-b_{1}}{a_{1}}, \frac{x_{2}-b_{2}}{a_{2}}, \ldots, \frac{x_{n}-b_{n}}{a_{n}}\right) d x_{1} d x_{2} \ldots d x_{n} \\
& =\int_{\mathbb{R}^{n}} \frac{f\left(x_{1}, x_{2}, \ldots, x_{n}\right)}{\sqrt{\left|a_{1} a_{2} \ldots a_{n}\right|}}\left|a_{1} a_{2} \ldots a_{n}\right| e^{-i\left(\lambda_{1} x_{1}+\lambda_{2} x_{2}+\cdots+\lambda_{n} x_{n}\right)} \\
& \times \overline{\hat{\psi}}\left(a_{1} \lambda_{1}, a_{2} \lambda_{2}, \ldots, a_{n} \lambda_{n}\right) d x_{1} d x_{2} \ldots d x_{n} \\
& =\prod_{i=1}^{n}\left|2 \pi a_{i}\right|^{1 / 2} \hat{f}\left(\lambda_{1}, \lambda_{2}, \ldots, \lambda_{n}\right) \overline{\hat{\psi}}\left(a_{1} \lambda_{1}, a_{2} \lambda_{2}, \ldots, a_{n} \lambda_{n}\right) .
\end{aligned}
$$


So

$$
\begin{aligned}
& F_{b_{1}, b_{2}, \ldots, b_{n}} W_{f}\left(a_{1}, a_{2}, \ldots, a_{n}, b_{1}, b_{2}, \ldots, b_{n}\right)\left(\lambda_{1}, \lambda_{2}, \ldots, \lambda_{n}\right) \\
& =\prod_{i=1}^{n} \sqrt{2 \pi\left|a_{i}\right|} \overline{\hat{\psi}}\left(a_{1} \lambda_{1}, a_{2} \lambda_{2}, \ldots, a_{n} \lambda_{n}\right) \hat{f}\left(\lambda_{1}, \lambda_{2}, \ldots, \lambda_{n}\right) .
\end{aligned}
$$

Step C. Final derivation of the inversion formula. Now take

$$
\begin{aligned}
F(x)=\frac{1}{C_{\psi}} \int_{\mathbb{R}^{n}} \int_{\mathbb{R}^{n}}\left|a_{1} a_{2} \ldots a_{n}\right|^{-1 / 2} \psi\left(\frac{x_{1}-b_{1}}{a_{1}}, \frac{x_{2}-b_{2}}{a_{2}}, \ldots, \frac{x_{n}-b_{n}}{a_{n}}\right) \\
\quad \times W_{f}\left(a_{1}, a_{2}, \ldots, a_{n}, b_{1}, b_{2}, \ldots, b_{n}\right) d b_{1} d b_{2} \ldots d b_{n} \frac{d a_{1} d a_{2} \ldots d a_{n}}{\left|a_{1} a_{2} \ldots a_{n}\right|^{2}} .
\end{aligned}
$$

Using Plancherel's formula we get

$$
\begin{aligned}
F(x)= & \frac{1}{C_{\psi}} \int_{\mathbb{R}^{n}} \frac{d a_{1} d a_{2} \ldots d a_{n}}{\sqrt{\left|a_{1} a_{2} \ldots a_{n}\right|}\left(a_{1} a_{2} \ldots a_{n}\right)^{2}} \\
\times & \int_{\mathbb{R}^{n}} \frac{F_{b_{1}, b_{2}, \ldots, b_{n}} \psi\left(\frac{x_{1}-b_{1}}{a_{1}}, \frac{x_{2}-b_{2}}{a_{2}}, \ldots, \frac{x_{n}-b_{n}}{a_{n}}\right)}{\left(\lambda_{1}, \lambda_{2}, \ldots, \lambda_{n}\right)} \\
\times & F_{b_{1}, b_{2}, \ldots, b_{n}} W_{f}\left(a_{1}, a_{2}, \ldots, a_{n}, b_{1}, b_{2}, \ldots, b_{n}\right)\left(\lambda_{1}, \lambda_{2}, \ldots, \lambda_{n}\right) d \lambda_{1} d \lambda_{2} \ldots d \lambda_{n} \\
= & \frac{1}{C_{\psi}} \int_{\mathbb{R}^{n}} \frac{d a_{1} d a_{2} \ldots d a_{n}}{\sqrt{\left|a_{1} a_{2} \ldots a_{n}\right|}\left(a_{1} a_{2} \ldots a_{n}\right)^{2}}\left|a_{1} a_{2} \ldots a_{n}\right| \\
& \times \int_{\mathbb{R}^{n}}\left[e^{i\left(\lambda_{1} x_{1}+\lambda_{2} x_{2}+\ldots+\lambda_{n} x_{n}\right)} \hat{\psi}\left(a_{1} \lambda_{1}, a_{2} \lambda_{2}, \ldots, a_{n} \lambda_{n}\right)\right. \\
& \left.\times \hat{f}\left(\lambda_{1}, \lambda_{2}, \ldots, \lambda_{n}\right) \prod_{i=1}^{n} \sqrt{\left|2 \pi a_{i}\right|} \overline{\hat{\psi}}\left(a_{1} \lambda_{1}, a_{2} \lambda_{2}, \ldots, a_{n} \lambda_{n}\right)\right] d \lambda_{1} d \lambda_{2} \ldots d \lambda_{n} \\
= & \frac{1}{C_{\psi}}(2 \pi)^{n / 2} \int_{\mathbb{R}^{n}} \int_{\mathbb{R}^{n}} \frac{d a_{1} d a_{2} \ldots d a_{n}}{\left|a_{1} a_{2} \ldots a_{n}\right|} e^{i \lambda_{1} x_{1}+i \lambda_{2} x_{2}+\ldots+i \lambda_{n} x_{n}} \\
& \times\left|\hat{\psi}\left(a_{1} \lambda_{1}, a_{2} \lambda_{2}, \ldots, a_{n} \lambda_{n}\right)\right|^{2} \hat{f}\left(\lambda_{1}, \lambda_{2}, \ldots, \lambda_{n}\right) d \lambda_{1} d \lambda_{2} \ldots d \lambda_{n} \\
= & \frac{1}{C_{\psi}}(2 \pi)^{n} \int_{\mathbb{R}^{n}} \frac{\left|\hat{\psi}\left(a_{1} \lambda_{1}, a_{2} \lambda_{2}, \ldots, a_{n} \lambda_{n}\right)\right|^{2}}{\left|a_{1} a_{2} \ldots a_{n}\right|} d a_{1} d a_{2} \ldots d a_{n} \\
& \times \frac{1}{(2 \pi)^{n / 2}} \int_{\mathbb{R}^{n}} e^{i \lambda_{1} x_{1}+i \lambda 2 x_{2}+\cdots+i \lambda_{n} x_{n}} \hat{f}\left(\lambda_{1}, \lambda_{2}, \ldots, \lambda_{n}\right) d \lambda_{1} d \lambda_{2} \ldots d \lambda_{n} \\
= & \frac{C_{\psi}}{C_{\psi}} f\left(x_{1}, x_{2}, \ldots, x_{n}\right) \\
= & f\left(x_{1}, x_{2}, \ldots, x_{n}\right) .
\end{aligned}
$$

Using the transformation $a_{i} \lambda_{i}=u_{i}, i=1,2, \ldots, n$ one can show that

$$
\begin{aligned}
\int_{\mathbb{R}^{n}} & \frac{\left|\hat{\psi}\left(a_{1} \lambda_{1}, a_{2} \lambda_{2}, \ldots, a_{n} \lambda_{n}\right)\right|^{2}}{\left|a_{1} a_{2} \ldots a_{n}\right|} d a_{1} d a_{2} \ldots d a_{n} \\
& =\int_{\mathbb{R}^{n}} \frac{\left|\hat{\psi}\left(u_{1}, u_{2}, \ldots, u_{n}\right)\right|^{2}}{\left|u_{1} u_{2} \ldots u_{n}\right|} d u_{1} d u_{2} \ldots d u_{n} .
\end{aligned}
$$

The convergence here is in $L^{2}\left(\mathbb{R}^{n}\right)$. But at points of continuity of $f$ the convergence is in the pointwise sense in view of the Fourier inversion theorem we used in (4.10). 


\section{ACKNOWLEDGEMENT}

The authors express their gratefulness to Professor Mike Moore of the School of Mathematics and Statistics, Carleton University, Ottawa, who carefully went through the manuscript and gave us some valuable suggestions. The authors also express their gratefulness to a referee for his constructive criticisms.

\section{REFERENCES}

[1] N.I. Akhiezer and I.M. Glazman, Theory of linear operators in Hilbert space, Frederick Ungar Publishing Company, New York, 1966.

[2] Jean Pierre Antoine et al., Two-dimensional Wavelets and Their Relatives, Barnes and Noble, 17 Feb. 2006.

[3] Albert Bogess and Francis J. Narcowich, A First Course in Wavelets with Fourier Analysis, Prentice Hall, NJ, 2001.

[4] Charles K. Chui, An introduction to wavelets, Wavelet Analysis and its Applications, vol. 1, Academic Press, Inc., Boston, MA, 1992. MR1150048 (93f:42055)

[5] Ingrid Daubechies, Ten lectures on wavelets. June 1990. Mathematics Department, University of Lowell, MA.

[6] Fritz Keinert, Wavelets and multiwavelets, Studies in Advanced Mathematics, Chapman \& Hall/CRC, Boca Raton, FL, 2004. MR2035222 (2005b:42001)

[7] Yves Meyer, Wavelets and operators, Cambridge Studies in Advanced Mathematics, vol. 37, Cambridge University Press, Cambridge, 1992. Translated from the 1990 French original by D. H. Salinger. MR.1228209 (94f:42001)

[8] Martin J. Mohlenkamp and María Cristina Pereyra, Wavelets, their friends, and what they can do for you, EMS Series of Lectures in Mathematics, European Mathematical Society (EMS), Zürich, 2008. MR2438140 (2010g:42001)

[9] J. N. Pandey, The Hilbert transform of Schwartz distributions and applications, Pure and Applied Mathematics (New York), John Wiley \& Sons, Inc., New York, 1996. A WileyInterscience Publication. MR.1363489 (97e:44010)

[10] Jagdish N. Pandey, Pseudo-orthants as a generalisation of orthants, Analysis (Berlin) 34 (2014), no. 2, 133-142, DOI 10.1515/anly-2012-1186. MR3213528

[11] Ram Shankar Pathak, The wavelet transform, Atlantis Studies in Mathematics for Engineering and Science, vol. 4, Atlantis Press, Paris; World Scientific Publishing Co. Pte. Ltd., Hackensack, NJ, 2009. MR2582570 (2011d:42002)

[12] Gilbert Strang and Truong Nguyen, Wavelets and filter banks, Wellesley-Cambridge Press, Wellesley, MA, 1996. MR1411910(98b:94003)

School of Mathematics and Statistics, Carleton University, Ottawa, Canada

E-mail address: Jimpandey1936@gmail.com

Department of Mathematical Sciences, Indian Institute of Technology, DSt-Cims Banaras Hindu University, India

E-mail address: sk_upadhyay2001@yahoo.com 